\title{
New Class of Binomial Sums and Their Applications
}

\author{
Jovan Mikić \\ University of Banja Luka \\ Faculty of Technology \\ Bosnia and Herzegovina \\ jovan.mikic@tf .unibl.org
}

\begin{abstract}
We consider certain " $S$-sums" which are multiparametric generalizations $S\left(n, m ; a_{1}, \ldots, a_{l}\right)$ of sums of powers of binomial coefficients. A new class of binomial sums, associated to the $S$-sums, is introduced. Also, some recursions for new sums are presented and proved.

We present three applications of our new sums. The first application is for the divisibility, by the central binomial coefficient, of the alternating sum of powers of binomial coefficients. Our proof is given more simply then originally by Calkin (1998) who used modular properties.

The second application is for the following binomial sum $S: \mathbb{Z}_{>=0}^{l+2} \rightarrow \mathbb{Z}$,

$$
S\left(2 n, m, a_{1}, a_{2}, \ldots, a_{l}\right)=\sum_{k=0}^{2 n}(-1)^{k}\left(\begin{array}{c}
2 n \\
k
\end{array}\right)^{m} \prod_{i=1}^{l}\left(\begin{array}{c}
a_{i}+k \\
k
\end{array}\right)\left(\begin{array}{c}
a_{i}+2 n-k \\
2 n-k
\end{array}\right)
$$

which arises as a generalization of a known identity connected with a famous Dixon's formula. We claim that, if $m$ is a positive integer, then $S\left(2 n, m, a_{1}, a_{2}, \ldots, a_{l}\right)$ is divisible by $\left(\begin{array}{c}2 n \\ n\end{array}\right)$ and $\left(\begin{array}{c}a_{i}+n \\ n\end{array}\right)$ for all $i=1, \ldots, l$. We give a proof for the case $l=1$.

As a third application, we present an interesting connection between our new sums and one Theorem by Guo, Jouhet and Zeng.
\end{abstract}

\section{Introduction}

Let $m, n$, and $k$ be non-negative integers such that $m \geq 1$. We consider the sum

$$
S(n, m)=\sum_{k=0}^{n}\left(\begin{array}{l}
n \\
k
\end{array}\right)^{m} F(n, k)
$$

where $F(n, k)$ is an integer-valued function that depends only on $n$ and $k$.

The aim is to examine some divisibility properties of sums of the form $S(n, m)$. To do this, we introduce the notion of " $M$ sums".

DOI: https://doi.org/10.5592/CO/CCD.2020.05 
Definition 1. Let $n, j$, and $t$ be non-negative integers such that $j \leq\left\lfloor\frac{n}{2}\right\rfloor$. Then the $M$ sums for $S(n, m)$ are as follows:

$$
M_{S}(n, j, t)=\left(\begin{array}{c}
n-j \\
j
\end{array}\right) \sum_{v=0}^{n-2 j}\left(\begin{array}{c}
n-2 j \\
v
\end{array}\right)\left(\begin{array}{c}
n \\
j+v
\end{array}\right)^{t} F(n, j+v) .
$$

Obviously, for $m \geq 1$, the equation

$$
S(n, m)=M_{S}(n, 0, m-1)
$$

holds.

Hence, we can see the sum $M_{S}(n, j, m-1)$ as a generalization of the sum $S(n, m)$.

Let $n, j$, and $t$ be as in Definition 1 . We present our main theorem:

\section{Theorem 2.}

$$
M_{S}(n, j, t+1)=\left(\begin{array}{c}
n \\
j
\end{array}\right) \sum_{u=0}^{\left\lfloor\frac{n-2 j}{2}\right\rfloor}\left(\begin{array}{c}
n-j \\
u
\end{array}\right) M_{S}(n, j+u, t) .
$$

Theorem 2 gives a recursive definition of a $M_{S}$ sum.

For proving Theorem 2, we use a method of " $D$ sums".

\section{Background}

Recently, in our previous paper [8], the notion of " $D$ sums" was introduced.

Let $n, j$, and $t$ be as in Definition 1 ; and let $S$ be as in Eq. (1).

Then the $D$ sums for $S(n, m)$ are

$$
D_{S}(n, j, t)=\sum_{l=0}^{n-2 j}\left(\begin{array}{c}
n-j \\
l
\end{array}\right)\left(\begin{array}{c}
n-j \\
j+l
\end{array}\right)\left(\begin{array}{c}
n \\
j+l
\end{array}\right)^{t} F(n, j+l) .
$$

For $m \geq 2$, by Eq. (4), it follows that

$$
S(n, m)=D_{S}(n, 0, m-2) .
$$

Furthermore, $D$ sums satisfy the following two recurrence relations [8, Thm. 2, p. 2], [11, Eqns. (14) and (15), p. 4]:

$$
\begin{aligned}
D_{S}(n, j, t+1) & =\sum_{u=0}^{\left\lfloor\frac{n-2 j}{2}\right\rfloor}\left(\begin{array}{c}
n \\
j+u
\end{array}\right)\left(\begin{array}{c}
n-j \\
u
\end{array}\right) D_{S}(n, j+u, t), \\
D_{S}(n, j, 0) & =\sum_{u=0}^{\left\lfloor\frac{n-2 j}{2}\right\rfloor}\left(\begin{array}{c}
n-j \\
u
\end{array}\right)\left(\begin{array}{c}
n-j-u \\
j+u
\end{array}\right) \sum_{v=0}^{n-2 j-2 u}\left(\begin{array}{c}
n-2 j-2 u \\
v
\end{array}\right) F(n, j+u+v) .
\end{aligned}
$$


In 1998, Calkin [1, Thm. 1] proved that the alternating binomial sum $\sum_{k=0}^{2 n}(-1)^{k}\left(\begin{array}{c}2 n \\ k\end{array}\right)^{m}$ is divisible by $\left(\begin{array}{c}2 n \\ n\end{array}\right)$ for all non-negative integers $n$ and all positive integers $m$. In 2007, Guo, Jouhet, and Zeng proved, among other things, two generalizations of Calkin's result [6, Thm. 1.2, Thm. 1.3, p. 2].

The first application of $D$ sums [8, Section 8] was for proving Calkin's result [1, Thm. 1]. Also, by using $D$ sums, it was proved [8] that $\sum_{k=0}^{2 n}\left(\begin{array}{c}2 n \\ k\end{array}\right)^{m}|n-k|$ is divisible by $n\left(\begin{array}{c}2 n \\ n\end{array}\right)$ for all non-negative integers $n$ and all positive integers $m$.

By the same method, it was proved [11, Thm. 1] that $\sum_{k=0}^{2 n}(-1)^{k}\left(\begin{array}{c}2 n \\ k\end{array}\right)^{m}\left(\begin{array}{c}2 k \\ k\end{array}\right)\left(\begin{array}{c}4 n-2 k \\ 2 n-k\end{array}\right)$ is divisible by $\left(\begin{array}{c}2 n \\ n\end{array}\right)$ for all non-negative integers $n$ and all positive integers $m$. Furthermore, it was proved [11, Corollary 4] that $\sum_{k=0}^{2 n}(-1)^{k}\left(\begin{array}{c}2 n \\ k\end{array}\right)^{m} C_{k} C_{2 n-k}$ is divisible by $\left(\begin{array}{c}2 n \\ n\end{array}\right)$ for all non-negative integers $n$; and for all positive integers $m$.

Let $S(k, l)=\frac{\left(\begin{array}{c}2 k \\ k\end{array}\right)\left(\begin{array}{c}2 l \\ l\end{array}\right)}{\left(\begin{array}{c}k+l \\ k\end{array}\right)}$ denote the $k$-th super Catalan number of order $l$. Recently, it was proved [10], that $\sum_{k=0}^{2 n}(-1)^{k}\left(\begin{array}{c}2 n \\ k\end{array}\right)^{m} S(k, l) S(2 n-k, l)$ is divisible by $S(n, l)$ for all non-negative integers $n$ and $l$ and all positive integers $m$.

We will show that there is a close relationship between $M$-sums and $D$-sums.

\section{Proof of Theorem 2}

We need one auxiliary result.

We will use a symmetry of binomial coefficients and the well-known binomial identity

$$
\left(\begin{array}{l}
a \\
b
\end{array}\right)\left(\begin{array}{l}
b \\
c
\end{array}\right)=\left(\begin{array}{l}
a \\
c
\end{array}\right)\left(\begin{array}{l}
a-c \\
b-c
\end{array}\right)
$$

where $a, b, c$ are non-negative integers such that $a \geq b \geq c$.

Lemma 3. Let $n$ and $j$ be non-negative integers; and let $t$ be a positive integer. Then the following equation

$$
M_{S}(n, j, t)=\left(\begin{array}{l}
n \\
j
\end{array}\right) D_{S}(n, j, t-1)
$$

holds.

Proof. By Eq. (2), we know that

$$
M_{S}(n, j, t)=\sum_{v=0}^{n-2 j}\left(\begin{array}{c}
n-j \\
j
\end{array}\right)\left(\begin{array}{c}
n-2 j \\
v
\end{array}\right)\left(\begin{array}{c}
n \\
j+v
\end{array}\right)^{t} F(n, j+v) .
$$

Starting from Eq. (10), by using Eq. (8) and a symmetry of binomial coefficients, it is 
not hard to prove that:

$$
\begin{aligned}
M_{S}(n, j, t) & =\sum_{v=0}^{n-2 j}\left(\begin{array}{c}
n-j \\
n-2 j
\end{array}\right)\left(\begin{array}{c}
n-2 j \\
v
\end{array}\right)\left(\begin{array}{c}
n \\
j+v
\end{array}\right)^{t} F(n, j+v) \\
& =\left(\begin{array}{c}
n \\
j
\end{array}\right) \sum_{v=0}^{n-2 j}\left(\begin{array}{c}
n-j \\
v
\end{array}\right)\left(\begin{array}{c}
n-j \\
j+v
\end{array}\right)\left(\begin{array}{c}
n \\
j+v
\end{array}\right)^{t-1} F(n, j+v) .
\end{aligned}
$$

By the definition of $D$ sums (see Eq. (4)) and the last equation above, we conclude that

$$
M_{S}(n, j, t)=\left(\begin{array}{c}
n \\
j
\end{array}\right) D_{S}(n, j, t-1) .
$$

This completes the proof of Lemma 3.

Now, we are ready for the proof of main theorem 2. We will use Eqns. (6), (7), and Lemma 3.

By Eq. (6), it follows that

$$
D_{S}(n, j, t+1)=\sum_{u=0}^{\left\lfloor\frac{n-2 j}{2}\right\rfloor}\left(\begin{array}{c}
n-j \\
u
\end{array}\right)\left(\begin{array}{c}
n \\
j+u
\end{array}\right) D_{S}(n, j+u, t) .
$$

Let us recall that $t$ in Eq. (11) is a non-negative integer.

By Lemma 3, it follows that

$$
\left(\begin{array}{c}
n \\
j+u
\end{array}\right) D_{S}(n, j+u, t)=M_{S}(n, j+u, t+1) .
$$

By Eq. (12), Eq. (11) becomes as follows:

$$
D_{S}(n, j, t+1)=\sum_{u=0}^{\left\lfloor\frac{n-2 j}{2}\right\rfloor}\left(\begin{array}{c}
n-j \\
u
\end{array}\right) M_{S}(n, j+u, t+1) .
$$

By multiplication of both sides of Eq. (13) with $\left(\begin{array}{l}n \\ j\end{array}\right)$, it follows that

$$
\left(\begin{array}{c}
n \\
j
\end{array}\right) D_{S}(n, j, t+1)=\left(\begin{array}{c}
n \\
j
\end{array}\right) \sum_{u=0}^{\left\lfloor\frac{n-2 j}{2}\right\rfloor}\left(\begin{array}{c}
n-j \\
u
\end{array}\right) M_{S}(n, j+u, t+1)
$$

By Lemma 3, Eq. (14) becomes as follows:

$$
M_{S}(n, j, t+2)=\left(\begin{array}{c}
n \\
j
\end{array}\right) \sum_{u=0}^{\left\lfloor\frac{n-2 j}{2}\right\rfloor}\left(\begin{array}{c}
n-j \\
u
\end{array}\right) M_{S}(n, j+u, t+1)
$$


By setting $t:=t+1$, the last equation above becomes

$$
M_{S}(n, j, t+1)=\left(\begin{array}{c}
n \\
j
\end{array}\right) \sum_{u=0}^{\left\lfloor\frac{n-2 j}{2}\right\rfloor}\left(\begin{array}{c}
n-j \\
u
\end{array}\right) M_{S}(n, j+u, t)
$$

where, now, $t$ is a positive integer.

Therefore, Theorem 2 follows from Eq. (15), if $t$ is a positive integer.

For $t=0$, we will use Eq. (7).

By Eq. (7), it follows that

$$
\begin{aligned}
D_{S}(n, j, 0) & =\sum_{u=0}^{\left\lfloor\frac{n-2 j}{2}\right\rfloor}\left(\begin{array}{c}
n-j \\
u
\end{array}\right)\left(\begin{array}{c}
n-(j+u) \\
j+u
\end{array}\right) \sum_{v=0}^{n-2(j+u)}\left(\begin{array}{c}
n-2(j+u) \\
v
\end{array}\right) F(n,(j+u)+v) \\
& =\sum_{u=0}^{\left\lfloor\frac{n-2 j}{2}\right\rfloor}\left(\begin{array}{c}
n-j \\
u
\end{array}\right) M_{S}(n, j+u, 0) .
\end{aligned}
$$

By the last equation above, it follows that

$$
D_{S}(n, j, 0)=\sum_{u=0}^{\left\lfloor\frac{n-2 j}{2}\right\rfloor}\left(\begin{array}{c}
n-j \\
u
\end{array}\right) M_{S}(n, j+u, 0) .
$$

By multiplication of both sides of Eq. (16) with $\left(\begin{array}{l}n \\ j\end{array}\right)$, it follows that

$$
\left(\begin{array}{l}
n \\
j
\end{array}\right) D_{S}(n, j, 0)=\left(\begin{array}{c}
n \\
j
\end{array}\right) \sum_{u=0}^{\left\lfloor\frac{n-2 j}{2}\right\rfloor}\left(\begin{array}{c}
n-j \\
u
\end{array}\right) M_{S}(n, j+u, 0) .
$$

By Lemma 3, we know that

$$
M_{S}(n, j, 1)=\left(\begin{array}{l}
n \\
j
\end{array}\right) D_{S}(n, j, 0) .
$$

By using the equation above, Eq. (17) becomes, as follows:

$$
M_{S}(n, j, 1)=\left(\begin{array}{c}
n \\
j
\end{array}\right) \sum_{u=0}^{\left\lfloor\frac{n-2 j}{2}\right\rfloor}\left(\begin{array}{c}
n-j \\
u
\end{array}\right) M_{S}(n, j+u, 0) .
$$

It is readily verified that Eq. (18) proves Theorem 2 for the case $t=0$.

Hence, Eqns. (15) and (18) complete the proof of Theorem 2. 
Remark 4. By Eqns. (13) and (16), it follows that

$$
D_{S}(n, j, t)=\sum_{u=0}^{\left\lfloor\frac{n-2 j}{2}\right\rfloor}\left(\begin{array}{c}
n-j \\
u
\end{array}\right) M_{S}(n, j+u, t)
$$

where $t$ is a non-negative integer.

Eqns. (9) and (19) give connections between $M$ and $D$ sums.

Note that Theorem 2 can be proved without using $D$ sums. See [8, Proof of Theorem 3, p. 6].

\section{How do we use "M" sums?}

In one particular situation, Theorem 2 implies a simple consequence which is important for us.

Let $n$ be a fixed non-negative integer. Let $t_{0}$ be a non-negative integer, and let $j$ be an arbitrary integer in the range $0 \leq j \leq\left\lfloor\frac{n}{2}\right\rfloor$. Suppose that $q=q(n)$ is a positive integer which divides $M_{S}\left(n, j, t_{0}\right)$ sums for all $j$ in the given range. We want $q$ to be as large as possible. Then it can be shown, by Theorem 2 , that $q$ divides $M_{S}\left(n, j, t_{0}+1\right)$ for all $j$ in the given range.

By induction, it follows that $q$ divides $M_{S}(n, j, t)$ for all $t$ such that $t \geq t_{0}$ and for all $j$ in the given range. By Eq. (3), it follows that $q$ divides $S(n, t+1)$ for all $t$ such that $t \geq t_{0}$.

See also [11, Section 5, p. 7].

\section{The first application for the alternating sum}

We give a proof of Calkin's result [1, Thm. 1] by using $M$ sums.

Let $n$ be a non-negative integer and let $m$ be a positive integer. Let

$$
S_{1}(2 n, m)=\sum_{k=0}^{2 n}(-1)^{k}\left(\begin{array}{c}
2 n \\
k
\end{array}\right)^{m} .
$$

Obviously, the sum $S_{1}(2 n, m)$ is an instance of the sum $(1)$, where $F_{1}(2 n, k)=(-1)^{k}$.

We use the well-known identity [2, Eq. (1.25), p. 4]

$$
\sum_{k=0}^{2 n}(-1)^{k}\left(\begin{array}{c}
2 n \\
k
\end{array}\right)= \begin{cases}0, & \text { if } n>0 \\
1, & \text { if } n=0\end{cases}
$$

where $n$ is a non-negative integer.

By Eq. (20), we conclude that $S_{1}(2 n, 1)$ is divisible by $\left(\begin{array}{c}2 n \\ n\end{array}\right)$. 
Furthermore, it is known

$$
\begin{array}{ll}
S_{1}(2 n, 2)=(-1)^{n}\left(\begin{array}{c}
2 n \\
n
\end{array}\right), & \text { (Kummer's formula) } \\
S_{1}(2 n, 3)=(-1)^{n}\left(\begin{array}{c}
2 n \\
n
\end{array}\right)\left(\begin{array}{c}
3 n \\
2 n
\end{array}\right) . & \text { (Dixon's formula) }
\end{array}
$$

Therefore, it follows that $S_{1}(2 n, m)$ is divisible by $\left(\begin{array}{c}2 n \\ n\end{array}\right)$ for $1 \leq m \leq 3$.

Let us calculate $M_{S_{1}}(2 n, j, 0)$ sum; where $j$ is a non-negative integer such that $j \leq n$.

Without loss of generality, let us assume that $n$ is a positive integer.

By Def. (1), it follows that

$$
\begin{aligned}
M_{S_{1}}(2 n, j, 0) & =\left(\begin{array}{c}
2 n-j \\
j
\end{array}\right) \sum_{v=0}^{2 n-2 j}\left(\begin{array}{c}
2 n-2 j \\
v
\end{array}\right)\left(\begin{array}{c}
2 n \\
j+v
\end{array}\right)^{0} F_{1}(2 n, j+v) \\
& =\left(\begin{array}{c}
2 n-j \\
j
\end{array}\right) \sum_{v=0}^{2 n-2 j}\left(\begin{array}{c}
2 n-2 j \\
v
\end{array}\right)(-1)^{j+v}
\end{aligned}
$$

By the last equation above, it follows that

$$
M_{S_{1}}(2 n, j, 0)=(-1)^{j}\left(\begin{array}{c}
2 n-j \\
j
\end{array}\right) \sum_{v=0}^{2(n-j)}(-1)^{v}\left(\begin{array}{c}
2(n-j) \\
v
\end{array}\right)
$$

By Eqns. (20) and (21), it follows that

$$
M_{S_{1}}(2 n, j, 0)= \begin{cases}0, & \text { if } 0 \leq j<n \\ (-1)^{n}, & \text { if } j=n\end{cases}
$$

Let us calculate $M_{S_{1}}(2 n, j, 1)$ sum. We will use Theorem 2 and Eq. (22). By setting $t=0$ in Theorem 2, it follows that

$$
M_{S_{1}}(2 n, j, 1)=\left(\begin{array}{c}
2 n \\
j
\end{array}\right) \sum_{u=0}^{n-j}\left(\begin{array}{c}
2 n-j \\
u
\end{array}\right) M_{S_{1}}(2 n, j+u, 0) .
$$

By Eq. (22), it follows that

$$
M_{S_{1}}(2 n, j+u, 0)= \begin{cases}0, & \text { if } 0 \leq u<n-j \\ (-1)^{n}, & \text { if } u=n-j\end{cases}
$$


By Eq. (24), Eq. (23) becomes gradually

$$
\begin{array}{rlrl}
M_{S_{1}}(2 n, j, 1) & =(-1)^{n}\left(\begin{array}{c}
2 n \\
j
\end{array}\right)\left(\begin{array}{c}
2 n-j \\
n-j
\end{array}\right) & \\
& =(-1)^{n}\left(\begin{array}{c}
2 n \\
2 n-j
\end{array}\right)\left(\begin{array}{c}
2 n-j \\
n
\end{array}\right) & & \text { (by symmetry) } \\
& =(-1)^{n}\left(\begin{array}{c}
2 n \\
n
\end{array}\right)\left(\begin{array}{c}
n \\
n-j
\end{array}\right) & & \text { (by Eq. (8)). }
\end{array}
$$

By the lst equation above, it follows that

$$
M_{S_{1}}(2 n, j, 1)=(-1)^{n}\left(\begin{array}{c}
2 n \\
n
\end{array}\right)\left(\begin{array}{c}
n \\
j
\end{array}\right)
$$

By setting $j=0$ in Eq. (25) and by using Eq. (3), we obtain Kummer's formula.

Furthermore, Eq. (25) suggets setting $q_{1}(2 n)=\left(\begin{array}{c}2 n \\ n\end{array}\right)$.

By Theorem 2 and induction, it can be shown that $M_{S_{1}}(2 n, j, t)$ is divisible by $\left(\begin{array}{c}2 n \\ n\end{array}\right)$ for all positive integers $t$ and all integers $j$ such that $0 \leq j \leq n$.

Let $m \geq 2$. By Eq. (3), we know that

$$
S_{1}(2 n, m)=M_{S_{1}}(2 n, 0, m-1) .
$$

Since $m-1 \geq 1$, the sum $M_{S_{1}}(2 n, 0, m-1)$ is divisible by $\left(\begin{array}{c}2 n \\ n\end{array}\right)$. By Eq. (3), $S_{1}(2 n, m)$ is divisible by $\left(\begin{array}{c}2 n \\ n\end{array}\right)$ for $m \geq 2$.

This proves Calkin's result.

Remark 5. By using $M$-sums, we also can prove Dixon's formula [5, 9]. Namely, by using Theorem 2, Eq. (25), and the Vandermonde identity, it can be shown that

$$
M_{S_{1}}(2 n, j, 2)=(-1)^{n}\left(\begin{array}{c}
2 n \\
n
\end{array}\right)\left(\begin{array}{c}
2 n \\
j
\end{array}\right)\left(\begin{array}{c}
3 n-j \\
2 n
\end{array}\right) .
$$

By setting $j=0$ in Eq. (26) and by using Eq. (3), we obtain Dixon's formula.

\section{A generalization of new sums}

We begin with the following definition:

Definition 6. Let $m, n, k, a_{1}, a_{2}, \ldots, a_{l}$ be non-negative integers such that $m \geq 1$. We consider the sum:

$$
S\left(n, m ; a_{1}, a_{2}, \ldots, a_{l}\right)=\sum_{k=0}^{n}\left(\begin{array}{l}
n \\
k
\end{array}\right)^{m} F\left(n, k ; a_{1}, a_{2}, \ldots, a_{l}\right),
$$

where $F\left(n, k ; a_{1}, a_{2}, \ldots, a_{l}\right)$ is an integer-valued function. 
The aim is to examine some divisibility properties of sums of the form $S\left(n, m, a_{1}, a_{2}, \ldots, a_{l}\right)$. To do this, we introduce a natural generalization of a notion of $M$ sums.

Definition 7. Let $n, j, t, a_{1}, a_{2}, \ldots, a_{l}$ be non-negative integers such that $j \leq\left\lfloor\frac{n}{2}\right\rfloor$. Then the $M$ sums for $S\left(n, m ; a_{1}, a_{2}, \ldots, a_{l}\right)$ are as follows:

$$
M_{S}\left(n, j, t ; a_{1}, a_{2}, \ldots, a_{l}\right)=\left(\begin{array}{c}
n-j \\
j
\end{array}\right) \sum_{v=0}^{n-2 j}\left(\begin{array}{c}
n-2 j \\
v
\end{array}\right)\left(\begin{array}{c}
n \\
j+v
\end{array}\right)^{t} F\left(n, j+v ; a_{1}, a_{2}, \ldots, a_{l}\right) .
$$

Obviously, for $m \geq 1$, the equation

$$
S\left(n, m ; a_{1}, a_{2}, \ldots, a_{l}\right)=M_{S}\left(n, 0, m-1 ; a_{1}, a_{2}, \ldots, a_{l}\right)
$$

holds.

Eqns. (28) and (29) are similar with Eqns. (2) and (3), respectively.

Let $n, j, t, a_{1}, a_{2}, \ldots, a_{l}$ be as in Definition 7 . Then the following theorem is true:

\section{Theorem 8.}

$$
M_{S}\left(n, j, t+1 ; a_{1}, a_{2}, \ldots, a_{l}\right)=\left(\begin{array}{c}
n \\
j
\end{array}\right) \sum_{u=0}^{\left\lfloor\frac{n-2 j}{2}\right\rfloor}\left(\begin{array}{c}
n-j \\
u
\end{array}\right) M_{S}\left(n, j+u, t ; a_{1}, a_{2}, \ldots, a_{l}\right) .
$$

The proof of Theorem 8 is similar with the proof of Theorem 2.

Remark 9. Similarly, we can introduce a natural generalization of a notion of $D$ sums. Let $n, j, t, a_{1}, a_{2}, \ldots, a_{l}$ be non-negative integers such that $j \leq\left\lfloor\frac{n}{2}\right\rfloor$. Then the $D$ sums for $S\left(n, m ; a_{1}, a_{2}, \ldots, a_{l}\right)$ are as follows:

$$
D_{S}\left(n, j, t ; a_{1}, a_{2}, \ldots, a_{l}\right)=\sum_{v=0}^{n-2 j}\left(\begin{array}{c}
n-j \\
v
\end{array}\right)\left(\begin{array}{c}
n-j \\
j+v
\end{array}\right)\left(\begin{array}{c}
n \\
j+v
\end{array}\right)^{t} F\left(n, j+v ; a_{1}, a_{2}, \ldots, a_{l}\right) .
$$

Obviously, for $m \geq 2$, the equation

$$
S\left(n, m ; a_{1}, a_{2}, \ldots, a_{l}\right)=D_{S}\left(n, 0, m-2 ; a_{1}, a_{2}, \ldots, a_{l}\right)
$$

holds.

Also, it can be readily verified that

$$
\begin{aligned}
D_{S}\left(n, j, t+1 ; a_{1}, a_{2}, \ldots a_{l}\right) & =\sum_{u=0}^{\left\lfloor\frac{n-2 j}{2}\right\rfloor}\left(\begin{array}{c}
n \\
j+u
\end{array}\right)\left(\begin{array}{c}
n-j \\
u
\end{array}\right) D_{S}\left(n, j+u, t ; a_{1}, a_{2}, \ldots a_{l}\right), \\
M_{S}\left(n, j, t ; a_{1}, a_{2}, \ldots a_{l}\right) & =\left(\begin{array}{c}
n \\
j
\end{array}\right) D_{S}\left(n, j, t-1 ; a_{1}, a_{2}, \ldots a_{l}\right), \\
D_{S}\left(n, j, t ; a_{1}, a_{2}, \ldots a_{l}\right) & =\sum_{u=0}^{\left\lfloor\frac{n-2 j}{2}\right\rfloor}\left(\begin{array}{c}
n-j \\
u
\end{array}\right) M_{S}\left(n, j+u, t ; a_{1}, a_{2}, \ldots a_{l}\right)
\end{aligned}
$$

Eqns. (31), (32), (33), and (34) are similar with Eqns. (5), (6), (9), and (19), respectively. 


\section{The second application of new sums}

Let us consider the following binomial sum $S: \mathbb{Z}_{>=0}^{l+2} \rightarrow \mathbb{Z}$

$$
S\left(2 n, m ; a_{1}, a_{2}, \ldots, a_{l}\right)=\sum_{k=0}^{2 n}(-1)^{k}\left(\begin{array}{c}
2 n \\
k
\end{array}\right)^{m} \prod_{i=1}^{l}\left(\begin{array}{c}
a_{i}+k \\
k
\end{array}\right)\left(\begin{array}{c}
a_{i}+2 n-k \\
2 n-k
\end{array}\right)
$$

where $l$ is a positive integer and $n, m, a_{1}, a_{2}, \ldots, a_{l}$ are non-negative integers.

For $m=1$ and $l=1$, the sum $S$ reduces to an interesting combinatorial identity. It is known [3, Eq. (6.56), p. 29] that

$$
\sum_{k=0}^{2 n}(-1)^{k}\left(\begin{array}{c}
2 n \\
k
\end{array}\right)\left(\begin{array}{c}
a+k \\
k
\end{array}\right)\left(\begin{array}{c}
a+2 n-k \\
2 n-k
\end{array}\right)=(-1)^{n}\left(\begin{array}{c}
2 n \\
n
\end{array}\right)\left(\begin{array}{c}
a+n \\
2 n
\end{array}\right) .
$$

By using Eq. (30), it follows that $S\left(2 n, 1, a_{1}\right)=(-1)^{n}\left(\begin{array}{c}2 n \\ n\end{array}\right)\left(\begin{array}{c}a_{1}+n \\ 2 n\end{array}\right)$.

Moreover, the following formula

$$
\sum_{k=0}^{2 n}(-1)^{k}\left(\begin{array}{c}
2 n \\
k
\end{array}\right)\left(\begin{array}{c}
a+k \\
k
\end{array}\right)\left(\begin{array}{c}
a+2 n-k \\
2 n-k
\end{array}\right)=\sum_{k=0}^{2 n}(-1)^{k}\left(\begin{array}{c}
2 n \\
k
\end{array}\right)\left(\begin{array}{l}
a \\
k
\end{array}\right)\left(\begin{array}{c}
a \\
2 n-k
\end{array}\right)
$$

holds. Note that the right-side of Eq. (36) is an instance of the well-known Dixon's formula [9]. Furthermore, the right-side of Eq. (36) has the following generalization:

$$
R\left(2 n, m, a_{1}, a_{2}, \ldots, a_{l}\right)=\sum_{k=0}^{2 n}(-1)^{k}\left(\begin{array}{c}
2 n \\
k
\end{array}\right)^{m} \prod_{i=1}^{l}\left(\begin{array}{c}
a_{i} \\
k
\end{array}\right)\left(\begin{array}{c}
a_{i} \\
2 n-k
\end{array}\right) .
$$

Remark 10. By using the "upper negation" [4, Eq. (5.14), p. 164], it follows that $S\left(2 n, m ; a_{1}, a_{2}, \ldots, a_{l}\right)=R\left(2 n, m ;-a_{1}-1, \ldots,-a_{l}-1\right)$. Hence, parameters $a_{1}, \ldots, a_{l}$ in the definition of the sum $S$ need not to be positive.

In 1998, Calkin [1, Thm. 1] proved that the alternating binomial sum $\sum_{k=0}^{2 n}(-1)^{k}\left(\begin{array}{c}2 n \\ k\end{array}\right)^{m}$ is divisible by $\left(\begin{array}{c}2 n \\ n\end{array}\right)$ for all non-negative integers $n$ and all positive integers $m$. It follows that $R(2 n, m, 2 n)$ is divisible by $\left(\begin{array}{c}2 n \\ n\end{array}\right)$ for all non-negative integers $n$ and $m$.

In 2007, Guo, Jouhet, and Zeng proved, among other things, two generalizations of Calkin's result $[6$, Thm. 1.2, Thm. 1.3, p. 2]. As a special case of [6, Thm. 1.2, p. 2], they gave a direct generalization of Calkin's result $[6$, Thm. 1.4, p. 8] which is as follows:

Theorem 11. Let $s$ be a positive integer greater than 1 , and let $k, n_{1}, n_{2}, \ldots, n_{s}$ be nonnegative integers. Then the sum

$$
\sum_{k=-n_{1}}^{n_{1}}(-1)^{k} \prod_{i=1}^{s}\left(\begin{array}{c}
n_{i}+n_{i+1} \\
n_{i}+k
\end{array}\right)
$$

is divisible by $\left(\begin{array}{c}n_{1}+n_{s} \\ n_{1}\end{array}\right)$, where $n_{s+1}=n_{1}$. 
By Theorem 11, it follows that the $\operatorname{sum} R\left(2 n, m, a_{1}, a_{2}, \ldots, a_{l}\right)$ is divisible by $\left(\begin{array}{c}2 n \\ n\end{array}\right)$ and by $\left(\begin{array}{c}a_{i} \\ n\end{array}\right)$ for all $i=1, l$. Take $s=m+2 l, n_{1}=n_{2}=\ldots=n_{m}=n$ and $n_{m+1}=a-n, n_{m+2}=n$, $\ldots n_{m+2 l-1}=a-n, n_{m+2 l}=n$ in Theorem 11 .

The sum $R\left(2 n, m, a_{1}, a_{2}, \ldots, a_{l}\right)$ is a generalization of the right-side of Eq. (36), while the sum $S\left(2 n, m, a_{1}, a_{2}, \ldots, a_{l}\right)$ is a generalization of the left-side of Eq. (36).

We claim that

Theorem 12. If $m$ is a positive integer, then $S\left(2 n, m, a_{1}, a_{2}, \ldots, a_{l}\right)$ is divisible by $\left(\begin{array}{c}2 n \\ n\end{array}\right)$ and $\left(\begin{array}{c}a_{i}+n \\ n\end{array}\right)$ for all $i=1, \ldots, l$.

Proof. We give a proof only for the case $l=1$. For the sake of brevity and clarity, the rest of the proof of Theorem 12 is omitted.

We consider the following sum

$$
S(2 n, m ; a)=\sum_{k=0}^{2 n}(-1)^{k}\left(\begin{array}{c}
2 n \\
k
\end{array}\right)^{m}\left(\begin{array}{c}
a+k \\
k
\end{array}\right)\left(\begin{array}{c}
a+2 n-k \\
2 n-k
\end{array}\right)
$$

where $a$ is a non-negative integer.

Obviously, the sum $S(2 n, m ; a)$ is an instance of Eq. (27); where $F(2 n, k ; a)=(-1)^{k}\left(\begin{array}{c}a+k \\ k\end{array}\right)\left(\begin{array}{c}a+2 n-k \\ 2 n-k\end{array}\right)$.

By Definition (7) and Eq. (28), it follows that

$$
M_{S}(2 n, j, 0 ; a)=\left(\begin{array}{c}
2 n-j \\
j
\end{array}\right) \sum_{v=0}^{2 n-2 j}(-1)^{v}\left(\begin{array}{c}
2 n-2 j \\
v
\end{array}\right)\left(\begin{array}{c}
a+j+v \\
j+v
\end{array}\right)\left(\begin{array}{c}
a+2 n-j-v \\
2 n-j-v
\end{array}\right) .
$$

By using substitution $v=k-j$, Eq. (38) becomes

$$
M_{S}(2 n, j, 0 ; a)=(-1)^{j}\left(\begin{array}{c}
2 n-j \\
j
\end{array}\right) \sum_{k=j}^{2 n-j}(-1)^{k}\left(\begin{array}{c}
2 n-2 j \\
k-j
\end{array}\right)\left(\begin{array}{c}
a+k \\
k
\end{array}\right)\left(\begin{array}{c}
a+2 n-k \\
2 n-k
\end{array}\right) .
$$

It is readily verified that (by Eq. (8))

$$
\left(\begin{array}{c}
2 n-j \\
j
\end{array}\right)\left(\begin{array}{c}
2 n-2 j \\
k-j
\end{array}\right)=\left(\begin{array}{c}
2 n-j \\
k-j
\end{array}\right)\left(\begin{array}{c}
2 n-k \\
2 n-k-j
\end{array}\right) .
$$

By using Eq. (40), Eq. (39) reduces to

$$
M_{S}(2 n, j, 0 ; a)=(-1)^{j} \sum_{k=j}^{2 n-j}(-1)^{k}\left(\begin{array}{c}
2 n-j \\
k-j
\end{array}\right)\left(\begin{array}{c}
2 n-k \\
2 n-k-j
\end{array}\right)\left(\begin{array}{c}
a+k \\
k
\end{array}\right)\left(\begin{array}{c}
a+2 n-k \\
2 n-k
\end{array}\right) .
$$

It is readily verified that (by Eq. (8))

$$
\left(\begin{array}{c}
a+2 n-k \\
2 n-k
\end{array}\right)\left(\begin{array}{c}
2 n-k \\
2 n-k-j
\end{array}\right)=\left(\begin{array}{c}
a+2 n-k \\
a+j
\end{array}\right)\left(\begin{array}{c}
a+j \\
j
\end{array}\right) .
$$


By using Eq. (42), Eq. (41) becomes

$$
M_{S}(2 n, j, 0 ; a)=(-1)^{j}\left(\begin{array}{c}
a+j \\
j
\end{array}\right) \sum_{k=j}^{2 n-j}(-1)^{k}\left(\begin{array}{c}
2 n-j \\
k-j
\end{array}\right)\left(\begin{array}{c}
a+k \\
a
\end{array}\right)\left(\begin{array}{c}
a+2 n-k \\
a+j
\end{array}\right) .
$$

Now we use a well-known Stanley's formula:

$$
\sum_{l=0}^{\min (m, n)}\left(\begin{array}{c}
a \\
m-l
\end{array}\right)\left(\begin{array}{c}
b \\
n-l
\end{array}\right)\left(\begin{array}{c}
a+b+l \\
l
\end{array}\right)=\left(\begin{array}{c}
a+n \\
m
\end{array}\right)\left(\begin{array}{c}
b+m \\
n
\end{array}\right) .
$$

Remark 13. The identity 44 is equivalent to the triple binomial identity

[4, Eq. (5.28), p. 171]; first proved by J. F. Pfaff [12]. See also [7, Ex. 31, p. 71] and [13, Problem 14, p. 4].

By Stanley's formula (44), it follows that

$$
\sum_{l=0}^{a}\left(\begin{array}{c}
2 n-k \\
a+j-l
\end{array}\right)\left(\begin{array}{c}
k-j \\
a-l
\end{array}\right)\left(\begin{array}{c}
2 n-j+l \\
l
\end{array}\right)=\left(\begin{array}{c}
a+2 n-k \\
a+j
\end{array}\right)\left(\begin{array}{c}
a+k \\
a
\end{array}\right) .
$$

By using Eq. (45), Eq. (43) reduces to:

$$
M_{S}(2 n, j, 0 ; a)=(-1)^{j}\left(\begin{array}{c}
a+j \\
j
\end{array}\right) \sum_{k=j}^{2 n-j}(-1)^{k}\left(\begin{array}{c}
2 n-j \\
k-j
\end{array}\right) \sum_{l=0}^{a}\left(\begin{array}{c}
2 n-k \\
a+j-l
\end{array}\right)\left(\begin{array}{c}
k-j \\
a-l
\end{array}\right)\left(\begin{array}{c}
2 n-j+l \\
l
\end{array}\right) .
$$

By changing the order of summation in the equation above, it follows that $M_{S}(2 n, j, 0 ; a)$ is equal to

$$
(-1)^{j}\left(\begin{array}{c}
a+j \\
j
\end{array}\right) \sum_{l=0}^{a}\left(\begin{array}{c}
2 n-j+l \\
l
\end{array}\right) \sum_{k=j+a-l}^{2 n-(j+a-l)}(-1)^{k}\left(\begin{array}{c}
2 n-j \\
k-j
\end{array}\right)\left(\begin{array}{c}
2 n-k \\
a+j-l
\end{array}\right)\left(\begin{array}{c}
k-j \\
a-l
\end{array}\right) .
$$

It is readily verified that (by Eq. (8))

$$
\left(\begin{array}{c}
2 n-j \\
k-j
\end{array}\right)\left(\begin{array}{c}
2 n-k \\
a+j-l
\end{array}\right)=\left(\begin{array}{c}
2 n-j \\
a+j-l
\end{array}\right)\left(\begin{array}{c}
2 n-2 j-a+l \\
k-j
\end{array}\right) .
$$

By using Eqns. (46) and (47), it follows that $M_{S}(2 n, j, 0 ; a)$ is equal to

$$
(-1)^{j}\left(\begin{array}{c}
a+j \\
j
\end{array}\right) \sum_{l=0}^{a}\left(\begin{array}{c}
2 n-j+l \\
l
\end{array}\right)\left(\begin{array}{c}
2 n-j \\
a+j-l
\end{array}\right) \sum_{k=j+a-l}^{2 n-(j+a-l)}(-1)^{k}\left(\begin{array}{c}
2 n-2 j-a+l \\
k-j
\end{array}\right)\left(\begin{array}{l}
k-j \\
a-l
\end{array}\right) .
$$

We know that

$$
\left(\begin{array}{c}
2 n-2 j-a+l \\
k-j
\end{array}\right)\left(\begin{array}{c}
k-j \\
a-l
\end{array}\right)=\left(\begin{array}{c}
2 n-2 j-a+l \\
a-l
\end{array}\right)\left(\begin{array}{c}
2 n-2 j-2 a+2 l \\
k-j-a+l
\end{array}\right) .
$$


By using the last two equation above, it follows that $M_{S}(2 n, j, 0 ; a)$ is equal to $(-1)^{j}\left(\begin{array}{c}a+j \\ j\end{array}\right) \sum_{l=0}^{a}\left(\begin{array}{c}2 n-j+l \\ l\end{array}\right)\left(\begin{array}{c}2 n-j \\ a+j-l\end{array}\right)\left(\begin{array}{c}2 n-2 j-a+l \\ a-l\end{array}\right) \sum_{k=j+a-l}^{2 n-(j+a-l)}(-1)^{k}\left(\begin{array}{c}2 n-2 j-2 a+2 l \\ k-j-a+l\end{array}\right)$.

Note that (by Eq. (8))

$$
\left(\begin{array}{c}
2 n-j \\
a+j-l
\end{array}\right)\left(\begin{array}{c}
2 n-2 j-a+l \\
a-l
\end{array}\right)=\left(\begin{array}{c}
2 n-j \\
a-l
\end{array}\right)\left(\begin{array}{c}
2 n-j-a+l \\
j+a-l
\end{array}\right) .
$$

By using the last two equation above, it follows that $M_{S}(2 n, j, 0 ; a)$ is equal to

$$
(-1)^{j}\left(\begin{array}{c}
a+j \\
j
\end{array}\right) \sum_{l=0}^{a}\left(\begin{array}{c}
2 n-j+l \\
l
\end{array}\right)\left(\begin{array}{c}
2 n-j \\
a-l
\end{array}\right)\left(\begin{array}{c}
2 n-j-a+l \\
j+a-l
\end{array}\right) \sum_{k=j+a-l}^{2 n-(j+a-l)}(-1)^{k}\left(\begin{array}{c}
2 n-2 j-2 a+2 l \\
k-j-a+l
\end{array}\right) .
$$

By Eq. (20), it follows that

$$
\sum_{k=j+a-l}^{2 n-j-a+l}(-1)^{k}\left(\begin{array}{c}
2 n-2 j-2 a+2 l \\
k-j-a+l
\end{array}\right)= \begin{cases}0, & \text { if } n-j-a+l>0 \\
(-1)^{j+a-l}, & \text { if } n-j-a+l=0\end{cases}
$$

By Eq. (49), it follows that only non-vanishing term in Eq. (48) is for $l=a+j-n$. Therefore, by Eq. (48), it follows that $M_{S}(2 n, j, 0 ; a)$ is equal to

$$
(-1)^{n-j}\left(\begin{array}{c}
a+j \\
j
\end{array}\right)\left(\begin{array}{c}
2 n-j+(a+j-n) \\
a+j-n
\end{array}\right)\left(\begin{array}{c}
2 n-j \\
a-(a+j-n)
\end{array}\right)\left(\begin{array}{c}
2 n-j-a+(a+j-n) \\
j+a-(a+j-n)
\end{array}\right) .
$$

From the last equation above and symmetry of binomial coefficients, it follows that

$$
M_{S}(2 n, j, 0 ; a)=(-1)^{n-j}\left(\begin{array}{c}
a+j \\
j
\end{array}\right)\left(\begin{array}{c}
a+n \\
2 n-j
\end{array}\right)\left(\begin{array}{c}
2 n-j \\
n
\end{array}\right) .
$$

Note that (by Eq. (8))

$$
\left(\begin{array}{c}
a+n \\
2 n-j
\end{array}\right)\left(\begin{array}{c}
2 n-j \\
n
\end{array}\right)=\left(\begin{array}{c}
a+n \\
n
\end{array}\right)\left(\begin{array}{c}
a \\
n-j
\end{array}\right) .
$$

Therefore, by using Eq. (51), Eq. (50) reduces to

$$
M_{S}(2 n, j, 0 ; a)=(-1)^{n-j}\left(\begin{array}{c}
a+j \\
j
\end{array}\right)\left(\begin{array}{c}
a+n \\
a
\end{array}\right)\left(\begin{array}{c}
a \\
n-j
\end{array}\right) .
$$

Eq. (52) suggets setting $q_{2}(2 n, a)=\left(\begin{array}{c}a+n \\ a\end{array}\right)$. Obviously, $q_{2}(2 n, a)$ does not depend on $j$.

By Theorem 8 and induction, it can be shown that $M_{S}(2 n, j, t ; a)$ is divisible by $\left(\begin{array}{c}a+n \\ a\end{array}\right)$ for all non-negative integers $t$ and all integers $j$ such that $0 \leq j \leq n$. 
Let $m \geq 1$. By Eq. (29), we know that

$$
S(2 n, m ; a)=M_{S}(2 n, 0, m-1 ; a) .
$$

Since $m \geq 1$, the sum $M_{S}(2 n, 0, m-1 ; a)$ is divisible by $\left(\begin{array}{c}a+n \\ a\end{array}\right)$. By Eq. (29), $S(2 n, m ; a)$ is divisible by $\left(\begin{array}{c}a+n \\ a\end{array}\right)$ for all positive $m$.

Let us prove that $S(2 n, m ; a)$ is divisible by $\left(\begin{array}{c}2 n \\ n\end{array}\right)$ for all positive $m$ and for all non-negative integers $n$ and $a$.

By setting $j=0$ in Eq. (50) and by using Eq. (29), we obtain Eq. (35). It follows that $\left(\begin{array}{c}2 n \\ n\end{array}\right)$ divides $S(2 n, 1 ; a)$.

Let us calculate $M_{S}(2 n, j, 1 ; a)$ sum. We will use Theorem 8 and Eq. (50).

By setting $t=0$ in Theorem 8 , we obtain that

$$
M_{S}(2 n, j, 1 ; a)=\left(\begin{array}{c}
2 n \\
j
\end{array}\right) \sum_{u=0}^{n-j}\left(\begin{array}{c}
2 n-j \\
u
\end{array}\right) M_{S}(2 n, j+u, 0 ; a) .
$$

By using Eq. (50), it follows that

$$
M_{S}(2 n, j+u, 0 ; a)=(-1)^{n-j-u}\left(\begin{array}{c}
a+j+u \\
j+u
\end{array}\right)\left(\begin{array}{c}
a+n \\
2 n-j-u
\end{array}\right)\left(\begin{array}{c}
2 n-j-u \\
n
\end{array}\right) .
$$

It is readily verified that (by Eq. (8))

$$
\left(\begin{array}{c}
2 n \\
j
\end{array}\right)\left(\begin{array}{c}
2 n-j \\
u
\end{array}\right)=\left(\begin{array}{c}
2 n \\
2 n-j-u
\end{array}\right)\left(\begin{array}{c}
j+u \\
u
\end{array}\right)
$$

By the last equation above, Eq. (53) becomes

$$
M_{S}(2 n, j, 1 ; a)=\sum_{u=0}^{n-j}\left(\begin{array}{c}
2 n \\
2 n-j-u
\end{array}\right)\left(\begin{array}{c}
j+u \\
u
\end{array}\right) M_{S}(2 n, j+u, 0 ; a) .
$$

By Eqns. (54) and $(55), M_{S}(2 n, j, 1 ; a)$ is equal to

$$
\sum_{u=0}^{n-j}\left(\begin{array}{c}
2 n \\
2 n-j-u
\end{array}\right)\left(\begin{array}{c}
j+u \\
u
\end{array}\right)(-1)^{n-j-u}\left(\begin{array}{c}
a+j+u \\
j+u
\end{array}\right)\left(\begin{array}{c}
a+n \\
2 n-j-u
\end{array}\right)\left(\begin{array}{c}
2 n-j-u \\
n
\end{array}\right) .
$$

Note that (by Eq. (8))

$$
\left(\begin{array}{c}
2 n \\
2 n-j-u
\end{array}\right)\left(\begin{array}{c}
2 n-j-u \\
n
\end{array}\right)=\left(\begin{array}{c}
2 n \\
n
\end{array}\right)\left(\begin{array}{c}
n \\
j+u
\end{array}\right) .
$$

By using the last equation above, it follows that

$$
M_{S}(2 n, j, 1 ; a)=\left(\begin{array}{c}
2 n \\
n
\end{array}\right) \sum_{u=0}^{n-j}\left(\begin{array}{c}
n \\
j+u
\end{array}\right)\left(\begin{array}{c}
j+u \\
u
\end{array}\right)(-1)^{n-j-u}\left(\begin{array}{c}
a+j+u \\
j+u
\end{array}\right)\left(\begin{array}{c}
a+n \\
2 n-j-u
\end{array}\right)
$$


By Eq. (57), Theorem 8, and induction, it can be shown that $M_{S}(2 n, j, t ; a)$ is divisible by $\left(\begin{array}{c}2 n \\ n\end{array}\right)$ for all positive integers $t$ and all integers $j$ such that $0 \leq j \leq n$.

Let $m \geq 2$. By Eq. (29), we know that

$$
S(2 n, m ; a)=M_{S}(2 n, 0, m-1 ; a) .
$$

Since $m \geq 2$, the sum $M_{S}(2 n, 0, m-1 ; a)$ is divisible by $\left(\begin{array}{c}2 n \\ n\end{array}\right)$. By Eq. $(29), S(2 n, m ; a)$ is divisible by $\left(\begin{array}{c}2 n \\ n\end{array}\right)$ for all positive $m$ and for all non-negative integers $n$ and $a$.

\section{The third application of new sums}

There is an interesting connection between Theorem 11 and $M$-sums.

We assert that the following implication is true:

Theorem 14. Let $s$ be a fixed positive integer greater than 2 , and let $k, n_{1}, n_{2}, \ldots, n_{s}$ be arbitrary non-negative integers. Let us suppose that the sum

$$
\sum_{k=-n_{1}}^{n_{1}}(-1)^{k} \prod_{i=1}^{s}\left(\begin{array}{c}
n_{i}+n_{i+1} \\
n_{i}+k
\end{array}\right)
$$

is divisible by $\left(\begin{array}{c}n_{1}+n_{s} \\ n_{1}\end{array}\right)$, where $n_{s+1}=n_{1}$. Then, by using $M$ sums and the assumption above, it follows that the following sum

$$
\sum_{k=-n_{1}}^{n_{1}}(-1)^{k}\left(\begin{array}{c}
2 n_{1} \\
k+n_{1}
\end{array}\right)^{m} \cdot \prod_{i=1}^{s-1}\left(\begin{array}{c}
n_{i}+n_{i+1} \\
n_{i}+k
\end{array}\right)
$$

is divisible by $\left(\begin{array}{c}2 n_{1} \\ n_{1}\end{array}\right),\left(\begin{array}{c}n_{1}+n_{2} \\ n_{1}\end{array}\right), \ldots,\left(\begin{array}{c}n_{s-2}+n_{s-1} \\ n_{s-2}\end{array}\right)$, and $\left(\begin{array}{c}n_{1}+n_{s-1} \\ n_{1}\end{array}\right)$; where $m$ is an arbitrary positive integer and $n_{s}=n_{1}$.

Proof. For $m=1$, the proof of Theorem 14 directly follows from our assumption.

Let us suppose that $m \geq 2$.

Let $P\left(2 n_{1}, m ; n_{2}, \ldots, n_{s-1}\right)$ denote $\sum_{k=-n_{1}}^{n_{1}}(-1)^{k}\left(\begin{array}{c}2 n_{1} \\ k+n_{1}\end{array}\right)^{m} \cdot \prod_{i=1}^{s-1}\left(\begin{array}{c}n_{i}+n_{i+1} \\ n_{i}+k\end{array}\right)$.

By using substitution $u=k+n_{1}$, it follows that

$$
P\left(2 n_{1}, m ; n_{2}, \ldots, n_{s-1}\right)=\sum_{u=0}^{2 n_{1}}(-1)^{u-n_{1}}\left(\begin{array}{c}
2 n_{1} \\
u
\end{array}\right)^{m} \cdot \prod_{i=1}^{s-1}\left(\begin{array}{c}
n_{i}+n_{i+1} \\
u+n_{i}-n_{1}
\end{array}\right)
$$

Obviously, the sum $P\left(2 n_{1}, m ; n_{2}, \ldots, n_{s-1}\right)$ is an instance of the sum from Definition 6 ; where

$$
F\left(2 n_{1}, u ; n_{2}, \ldots, n_{s-1}\right)=(-1)^{u-n_{1}} \prod_{i=1}^{s-1}\left(\begin{array}{c}
n_{i}+n_{i+1} \\
u+n_{i}-n_{1}
\end{array}\right) .
$$


By using Definition 7 and Eq. (28), we obtain that

$M_{P}\left(2 n_{1}, j, 0 ; n_{2}, \ldots n_{s-1}\right)=\left(\begin{array}{c}2 n_{1}-j \\ j\end{array}\right) \sum_{v=0}^{2 n_{1}-2 j}\left(\begin{array}{c}2 n_{1}-2 j \\ v\end{array}\right) F\left(2 n_{1}, j+v ; n_{2}, \ldots n_{s-1}\right)$.

By using substitution $u=v+j$, the Eq. (59) reduces to

$$
M_{P}\left(2 n_{1}, j, 0 ; n_{2}, \ldots n_{s-1}\right)=\left(\begin{array}{c}
2 n_{1}-j \\
j
\end{array}\right) \sum_{u=j}^{2 n_{1}-j}\left(\begin{array}{c}
2 n_{1}-2 j \\
u-j
\end{array}\right) F\left(2 n_{1}, u ; n_{2}, \ldots n_{s-1}\right) .
$$

By using Eq. (58), it follows that $M_{P}\left(2 n_{1}, j, 0 ; n_{2}, \ldots n_{s-1}\right)$ is equal to the following sum

$$
\left(\begin{array}{c}
2 n_{1}-j \\
j
\end{array}\right) \sum_{u=j}^{2 n_{1}-j}\left(\begin{array}{c}
2 n_{1}-2 j \\
u-j
\end{array}\right)(-1)^{u-n_{1}} \prod_{i=1}^{s-1}\left(\begin{array}{c}
n_{i}+n_{i+1} \\
u+n_{i}-n_{1}
\end{array}\right)
$$

It is readily verified that (by Eq. (8))

$$
\left(\begin{array}{c}
2 n_{1}-j \\
j
\end{array}\right)\left(\begin{array}{c}
2 n_{1}-2 j \\
u-j
\end{array}\right)=\left(\begin{array}{c}
2 n_{1}-j \\
u-j
\end{array}\right)\left(\begin{array}{c}
2 n_{1}-u \\
j
\end{array}\right)
$$

By using Eqns. (61) and (62), it follows that $M_{P}\left(2 n_{1}, j, 0 ; n_{2}, \ldots n_{s-1}\right)$ is equal to

$$
\sum_{u=j}^{2 n_{1}-j}(-1)^{u-n_{1}}\left(\begin{array}{c}
2 n_{1}-j \\
u-j
\end{array}\right)\left(\begin{array}{c}
2 n_{1}-u \\
j
\end{array}\right) \prod_{i=1}^{s-1}\left(\begin{array}{c}
n_{i}+n_{i+1} \\
u+n_{i}-n_{1}
\end{array}\right)
$$

By a symmetry of binomial coefficients, we know that the last term in a product equals

$$
\left(\begin{array}{c}
n_{s-1}+n_{1} \\
u+n_{s-1}-n_{1}
\end{array}\right)=\left(\begin{array}{c}
n_{s-1}+n_{1} \\
2 n_{1}-u
\end{array}\right)
$$

The right-side of Eq. (63) can be rewritten as

$$
\sum_{u=j}^{2 n_{1}-j}(-1)^{u-n_{1}}\left(\begin{array}{c}
2 n_{1}-j \\
u-j
\end{array}\right)\left(\begin{array}{c}
n_{s-1}+n_{1} \\
2 n_{1}-u
\end{array}\right)\left(\begin{array}{c}
2 n_{1}-u \\
j
\end{array}\right) \prod_{i=1}^{s-2}\left(\begin{array}{c}
n_{i}+n_{i+1} \\
u+n_{i}-n_{1}
\end{array}\right)
$$

It is readily verified that (by Eq. (8))

$$
\left(\begin{array}{c}
n_{s-1}+n_{1} \\
2 n_{1}-u
\end{array}\right)\left(\begin{array}{c}
2 n_{1}-u \\
j
\end{array}\right)=\left(\begin{array}{c}
n_{s-1}+n_{1} \\
j
\end{array}\right)\left(\begin{array}{l}
n_{s-1}+n_{1}-j \\
u+n_{s-1}-n_{1}
\end{array}\right) .
$$

By using Eqns. (64) and (65), it follows that $M_{P}\left(2 n_{1}, j, 0 ; n_{2}, \ldots n_{s-1}\right)$ is equal to

$$
\left(\begin{array}{c}
n_{s-1}+n_{1} \\
j
\end{array}\right) \sum_{u=j}^{2 n_{1}-j}(-1)^{u-n_{1}}\left(\begin{array}{c}
n_{s-1}+n_{1}-j \\
u+n_{s-1}-n_{1}
\end{array}\right)\left(\begin{array}{c}
2 n_{1}-j \\
u-j
\end{array}\right) \prod_{i=1}^{s-2}\left(\begin{array}{c}
n_{i}+n_{i+1} \\
u+n_{i}-n_{1}
\end{array}\right) .
$$


By using substitution $p=u-n_{1}$ in Eq. (66), it follows that $M_{P}\left(2 n_{1}, j, 0 ; n_{2}, \ldots n_{s-1}\right)$ is equal to

$$
\left(\begin{array}{c}
n_{s-1}+n_{1} \\
j
\end{array}\right) \sum_{p=-\left(n_{1}-j\right)}^{n_{1}-j}(-1)^{p}\left(\begin{array}{c}
\left(n_{1}-j\right)+n_{1} \\
p+n_{1}-j
\end{array}\right)\left(\prod_{i=1}^{s-2}\left(\begin{array}{c}
n_{i}+n_{i+1} \\
p+n_{i}
\end{array}\right)\right)\left(\begin{array}{c}
n_{s-1}+\left(n_{1}-j\right) \\
p+n_{s-1}
\end{array}\right) .
$$

By using our assumption in Theorem 14, it follows that inner sum in Eq. (67) is divisible by integers $\left(\begin{array}{c}2 n_{1}-j \\ n_{1}\end{array}\right),\left(\begin{array}{c}n_{1}+n_{2} \\ n_{1}\end{array}\right), \ldots,\left(\begin{array}{c}n_{s-2}+n_{s-1} \\ n_{s-2}\end{array}\right)$, and by $\left(\begin{array}{c}n_{s-1}+n_{1}-j \\ n_{s-1}\end{array}\right)$.

Therefore, we obtain that $M_{P}\left(2 n_{1}, j, 0 ; n_{2}, \ldots n_{s-1}\right)$ is divisible by integers $\left(\begin{array}{c}2 n_{1}-j \\ n_{1}\end{array}\right),\left(\begin{array}{c}n_{1}+n_{2} \\ n_{1}\end{array}\right)$, $\ldots,\left(\begin{array}{c}n_{s-2}+n_{s-1} \\ n_{s-2}\end{array}\right)$, and by $\left(\begin{array}{c}n_{s-1}+n_{1}-j \\ n_{s-1}\end{array}\right)$ for all non-negative integers $j$ such that $j \leq n_{1}$.

By using Theorem 8 and the induction principle, it can be shown that $M_{P}\left(2 n_{1}, j, t ; n_{2}, \ldots n_{s-1}\right)$ is divisible by integers $\left(\begin{array}{c}n_{1}+n_{2} \\ n_{1}\end{array}\right), \ldots,\left(\begin{array}{c}n_{s-2}+n_{s-1} \\ n_{s-2}\end{array}\right)$ for all non-negative integers $t$ and $j$ such that $j \leq n_{1}$. By using Eq. (29), it follows that the sum $P\left(2 n_{1}, m ; n_{2}, \ldots, n_{s-1}\right)$ is divisible by integers $\left(\begin{array}{c}n_{1}+n_{2} \\ n_{1}\end{array}\right), \ldots,\left(\begin{array}{c}n_{s-2}+n_{s-1} \\ n_{s-2}\end{array}\right)$ for all positive integers $m$.

Let us prove that $P\left(2 n_{1}, m ; n_{2}, \ldots, n_{s-1}\right)$ is divisible by $\left(\begin{array}{c}2 n_{1} \\ n_{1}\end{array}\right)$ for all positive integers $m \geq 2$.

By setting $t=0$ in Theorem 8 , we obtain that

$$
M_{S}\left(2 n_{1}, j, 1 ; n_{2}, \ldots n_{s-1}\right)=\left(\begin{array}{c}
2 n_{1} \\
j
\end{array}\right) \sum_{u=0}^{n_{1}-j}\left(\begin{array}{c}
2 n_{1}-j \\
u
\end{array}\right) M_{S}\left(2 n_{1}, j+u, 0 ; n_{2}, \ldots n_{s-1}\right) .
$$

We know that $M_{S}\left(2 n_{1}, j+u, 0 ; n_{2}, \ldots n_{s-1}\right)=\left(\begin{array}{c}2 n_{1}-(j+u) \\ n_{1}\end{array}\right) \cdot c\left(j, u, n_{1}, \ldots, n_{s-1}\right)$; where $c\left(j, u, n_{1}, \ldots, n_{s-1}\right)$ is an integer.

By using Eq. (68), we have gradually

$$
\begin{aligned}
& M_{S}\left(2 n_{1}, j, 1 ; n_{2}, \ldots n_{s-1}\right)=\left(\begin{array}{c}
2 n_{1} \\
j
\end{array}\right) \sum_{u=0}^{n_{1}-j}\left(\begin{array}{c}
2 n_{1}-j \\
u
\end{array}\right)\left(\begin{array}{c}
2 n_{1}-j-u \\
n_{1}
\end{array}\right) \cdot c\left(j, u, n_{1}, \ldots, n_{s-1}\right) \\
& M_{S}\left(2 n_{1}, j, 1 ; n_{2}, \ldots n_{s-1}\right)=\sum_{u=0}^{n_{1}-j}\left(\begin{array}{c}
2 n_{1} \\
j
\end{array}\right)\left(\left(\begin{array}{c}
2 n_{1}-j \\
2 n_{1}-j-u
\end{array}\right)\left(\begin{array}{c}
2 n_{1}-j-u \\
n_{1}
\end{array}\right)\right) \cdot c\left(j, u, n_{1}, \ldots, n_{s-1}\right) \\
& M_{S}\left(2 n_{1}, j, 1 ; n_{2}, \ldots n_{s-1}\right)=\sum_{u=0}^{n_{1}-j}\left(\begin{array}{c}
2 n_{1} \\
j
\end{array}\right)\left(\left(\begin{array}{c}
2 n_{1}-j \\
n_{1}
\end{array}\right)\left(\begin{array}{c}
n_{1}-j \\
n_{1}-j-u
\end{array}\right)\right) \cdot c\left(j, u, n_{1}, \ldots, n_{s-1}\right) \\
& M_{S}\left(2 n_{1}, j, 1 ; n_{2}, \ldots n_{s-1}\right)=\sum_{u=0}^{n_{1}-j}\left(\left(\begin{array}{c}
2 n_{1} \\
2 n_{1}-j
\end{array}\right)\left(\begin{array}{c}
2 n_{1}-j \\
n_{1}
\end{array}\right)\right)\left(\begin{array}{c}
n_{1}-j \\
n_{1}-j-u
\end{array}\right) \cdot c\left(j, u, n_{1}, \ldots, n_{s-1}\right) \\
& M_{S}\left(2 n_{1}, j, 1 ; n_{2}, \ldots n_{s-1}\right)=\left(\begin{array}{c}
2 n_{1} \\
n_{1}
\end{array}\right) \sum_{u=0}^{n_{1}-j}\left(\begin{array}{c}
n_{1} \\
j
\end{array}\right)\left(\begin{array}{c}
n_{1}-j \\
u
\end{array}\right) \cdot c\left(j, u, n_{1}, \ldots, n_{s-1}\right) .
\end{aligned}
$$

By using the Eq. (69), it follows that $M_{S}\left(2 n_{1}, j, 1 ; n_{2}, \ldots n_{s-1}\right)$ is divisible by $\left(\begin{array}{c}2 n_{1} \\ n_{1}\end{array}\right)$ for all non-negative $j$ such that $j \leq n_{1}$. 
By using Eq. (29), it follows that the sum $P\left(2 n_{1}, m ; n_{2}, \ldots, n_{s-1}\right)$ is divisible by $\left(\begin{array}{c}2 n_{1} \\ n_{1}\end{array}\right)$ for all positive integers $m$ such that $m \geq 2$.

Finally, let us prove that $P\left(2 n_{1}, m ; n_{2}, \ldots, n_{s-1}\right)$ is divisible by $\left(\begin{array}{c}n_{1}+n_{s-1} \\ n_{1}\end{array}\right)$ for all positive integers $m$.

By Eq. (67) and our assumption, it follows that

$$
M_{P}\left(2 n_{1}, j, 0 ; n_{2}, \ldots n_{s-1}\right)=\left(\begin{array}{c}
n_{s-1}+n_{1} \\
j
\end{array}\right)\left(\begin{array}{c}
n_{1}-j+n_{s-1} \\
n_{s-1}
\end{array}\right) \cdot d\left(n_{1}-j, n_{2}, \ldots, n_{s-1}\right)
$$

where $d\left(n_{1}-j, n_{2}, \ldots, n_{s-1}\right)$ is an integer.

It is readily verified that (by Eq. (8))

$$
\left(\begin{array}{c}
n_{s-1}+n_{1} \\
j
\end{array}\right)\left(\begin{array}{c}
n_{1}-j+n_{s-1} \\
n_{s-1}
\end{array}\right)=\left(\begin{array}{c}
n_{1}+n_{s-1} \\
n_{s-1}
\end{array}\right)\left(\begin{array}{c}
n_{1} \\
j
\end{array}\right)
$$

By using Eq. (71), Eq. (70) becomes

$$
M_{P}\left(2 n_{1}, j, 0 ; n_{2}, \ldots n_{s-1}\right)=\left(\begin{array}{c}
n_{1}+n_{s-1} \\
n_{s-1}
\end{array}\right)\left(\begin{array}{c}
n_{1} \\
j
\end{array}\right) \cdot d\left(n_{1}-j, n_{2}, \ldots, n_{s-1}\right) .
$$

By using Theorem 8 and the induction principle, it can be shown that $M_{P}\left(2 n_{1}, j, t ; n_{2}, \ldots n_{s-1}\right)$ is divisible by an integer $\left(\begin{array}{c}n_{1}+n_{s-1} \\ n_{s-1}\end{array}\right)$ for all non-negative integers $t$ and $j$ such that $j \leq n_{1}$. By using Eq. (29), it follows that the sum $P\left(2 n_{1}, m ; n_{2}, \ldots, n_{s-1}\right)$ is divisible by an integer $\left(\begin{array}{c}n_{1}+n_{s-1} \\ n_{s-1}\end{array}\right)$ for all positive integers $m$.

This completes the proof of Theorem 14 .

\section{Acknowledgments}

I would like to thank to my aunt Bosiljka Djurdjević and uncle Čedo Stanić. Also I would like to thank the anonymous referee for valuable comments which helped to improve the article.

\section{References}

[1] N. J. Calkin, Factors of sums of powers of binomial coefficients, Acta Arith. 86 (1998), $17-26$.

[2] H. W. Gould, Combinatorial Identities, published by the author, revised edition, 1972.

[3] H. W. Gould and J. Quaintance, Combinatorial identities: Table I: intermediate techniques for summing finite series, preprint, 2010. Available at https://www. math. wvu . edu/ gould/Vol.4.PDF, 
[4] R. L. Graham, D. Knuth, and O. Patashnik, Concrete Mathematics, second edition.

[5] V. J. W. Guo, A simple proof of Dixon's identity, Discrete Math. 268 (2003), 309-310.

[6] V. J. W. Guo, F. Jouhet, and J. Zeng, Factors of alternating sums of products of binomial and q-binomial coefficients, Acta Arith. 127 (2007), 17-31.

[7] D. E. Knuth, The Art of Computer Programming, volume 1, third edition.

[8] J. Mikić, A method for examining divisibility properties of some binomial sums, $J$. Inteqer Sequences 21 (2018), Article 18.8.7.

[9] J. Mikić, A Proof of Dixon's Identity, J. Integer Sequences 19 (2016), Article 16.5.3.

[10] J. Mikić, On a New Alternating Convolution Formula for the Super Catalan Numbers, Romanian Mathematical Magazine (2021).

[11] J. Mikić, On Certain Sums Divisible by the Central Binomial Coefficient, J. Inteqer Sequences 23 (2020), Article 20.1.6.

[12] J. Pfaff, Observationes analyticae ad L. Euler Institutiones Calculi Integralis, Vol. IV, Supplem. II et IV, Historia de 1793, Nova Acta Acad. Sci. Petropolitanae, 11 (1797), pp. $38-57$.

[13] R. P. Stanley, Bijective proof problems (2009)

2010 Mathematics Subject Classification: Primary 05A10 ; Secondary 05A19.

Keywords: $M$ sum, method of $D$ sums, alternating binomial sum, recurrence relation, Stanley's formula, Dixon's formula. 
